\title{
Comparison of two computational methods for solvent screening in countercurrent and centrifugal partition chromatography
}

\author{
Sérgio M. Vilas-Boas ${ }^{a, b}$, Isabella W. Cordovaa,b,c, Kiki A. Kurnia ${ }^{\mathrm{d}}$, Heloísa H.S. Almeida ${ }^{a}$, \\ Priscilla S. Gaschi ${ }^{\text {c }}$, João A.P. Coutinho ${ }^{\text {b }}$, Simão P. Pinho ${ }^{a}$, Olga Ferreira ${ }^{\mathrm{a}, *}$ \\ ${ }^{a}$ Centro de Investigação de Montanha (CIMO), Instituto Politécnico de Bragança, Campus de Santa Apolónia, 5300-253 Bragança, Portugal \\ ${ }^{\mathrm{b}}$ CICECO - Aveiro Institute of Materials, Department of Chemistry, University of Aveiro, 3810-193 Aveiro, Portugal \\ ${ }^{c}$ UTFPR - Departamento de Engenharia Química, Universidade Tecnológica Federal do Paraná, 84016-210 Ponta Grossa, Brazil \\ ${ }^{\mathrm{d}}$ Department of Chemical Engineering, Faculty of Industrial Technology, Institut Teknologi Bandung, Bandung 40132, Indonesia
}

\section{A R T I C L E I N F O}

\section{Article history:}

Received 12 October 2021

Revised 24 January 2022

Accepted 25 January 2022

Available online 29 January 2022

\section{Keywords:}

Phenolic compounds

Countercurrent chromatography

Partition chromatography

Partition coefficients

NRTL-SAC

COSMO-RS

\begin{abstract}
A B S T R A C T
Countercurrent and centrifugal partition chromatography are techniques applied in the separation and isolation of compounds from natural extracts. One of the key design parameters of these processes is the selection of the biphasic solvent system that provides for the adequate partitioning of the solutes. To address this challenging task, the fully predictive Conductor-like Screening Model for Real Solvents (COSMO-RS) and the semi-predictive Non-Random Two-Liquid Segment Activity Coefficient (NRTL-SAC) model were applied to estimate the partition coefficients $(K)$ of four model phenolic compounds (vanillin, ferulic acid, $(S)$-hesperetin and quercetin) in different solvent systems. Complementing the experimental data collected in the literature, partition coefficients of each solute in binary, or quaternary, solvent systems were measured at $298.2 \mathrm{~K}$.

Higher deviations from the experimental data were obtained using the predictive COSMO-RS model, with an average RMSD (root-mean-square deviation) in $\log (K)$ of 1.17 of all four solutes (61 data points), providing a satisfactory quantitative description only for the systems containing vanillin (RSMD $=0.57$ ). For the NRTL-SAC model, the molecular parameters of the solutes were initially calculated by correlating a set of $K$ and solubility ( $x$, in mole fraction) data (16 partition coefficients and 44 solubility data points), for which average RMSD values of 0.07 and 0.41 were obtained in $\log (K)$ and $\log (x)$, respectively. The predictions of the remaining $\log (K)$ data (45 partition coefficients) resulted in an average RMSD of 0.43 , suggesting that the NRTL-SAC model was a more reliable quantitative solvent screening tool. Depending on the amount of available solubility and partition data, both models can be valuable alternatives in the preliminary stages of solvent screening destined to select the optimal mobile and stationary phases for a given separation.
\end{abstract}

(c) 2022 Elsevier B.V. All rights reserved.

\section{Introduction}

Natural matrices are a recognized source of valuable compounds with diverse structures and functionalities that find numerous applications in the pharmaceutical, food, chemical, and cosmetics industries [1]. The isolation of the target compounds usually starts with an extraction step, followed by several fractionation and purification processes to isolate them from the complex multicomponent mixtures forming the crude extracts [2]. This work focuses on separation techniques in which the solutes are trans-

\footnotetext{
* Corresponding author.

E-mail address: oferreira@ipb.pt (O. Ferreira).
}

ferred between two immiscible liquid phases in contact with each other [3]. As a first approach, simple solvent partitioning can be used to pre-concentrate families of compounds and, then, further purification can be achieved by chromatographic techniques such as countercurrent (CCC) and centrifugal partition chromatography (CPC) [2]. The separation principle of these chromatographic techniques relies on the sample partition between two immiscible stationary and mobile liquid phases. The main advantages compared to solid-liquid chromatographic methods are well known, and include the use of lower amounts of solvents, no loss of components due to adsorption onto a solid support, lower maintenance costs, and the possibility of scaling up the process to pilot and industrial levels [4,5]. Both CPC and CCC techniques have been extensively applied to medicinal plant and natural product research [6], from 
which relevant examples in the biorefinery context can be highlighted: the separation of monosaccharides from hydrolysed sugar beet pulp [7], the fractionation of phenolic acids [8], and the separation and purification of lignocellulosic biomass products (carbohydrates, furans, carboxylic acids and phenols) [9]. In this work, phenolics present in natural matrices are the target compounds; thus, vanillin (phenolic aldehyde), ferulic acid (hydroxycinnamic acid), (S)-hesperetin (flavanone), and quercetin (flavonol) were selected as model compounds.

In designing such processes, a crucial and time-consuming task is the selection of the optimum biphasic solvent system. It usually demands the experimental measurements of partition coefficients or relying on literature data for similar separation problems [10]. The number of possible combinations is almost infinite, but a few traditional families of solvent systems can be named, covering a wide range of polarities, as recently reviewed by Liu et al. [11]: Ito system [12] (hexane/ethyl acetate/methanol/1-butanol/water), Arizona system [13,14] (heptane/ethyl acetate/methanol/water), or HEMWat system [15] (hexane/ethyl acetate/methanol/water). Woźniak and Garrard [16] organized an extensive database by classifying 2594 solvent systems used for natural product purifications, dividing the solvents into 7 types and the solutes into 21 classes. Water, ethyl acetate, methanol, and hexane were the most used solvents, followed by 1-butanol and chloroform. Overall, alkane-based solvent systems (alkane is the primary water-immiscible solvent in the system, as defined by the authors) were the most commonly used, considering all classes of solutes, including flavonoids, phenolic acids, and phenol derivatives studied here [16]. A similar conclusion regarding flavonoids was also reported by Costa and Leitão [17], suggesting the HEMWat solvent system as a first approach for the separation of samples containing free flavonoids, and the more polar ternary system composed of ethyl acetate/butanol/water, for the isolation of glycosylated flavonoids. Besides these traditional biphasic systems, a more sustainable alternative to alkane-based solvent systems was proposed in the literature. Faure et al. [18,19] studied a green version of the Arizona system, in which the less toxic ethanol replaced methanol, and limonene, a biorenewable cycloterpene, replaced heptane, and reported the physico-chemical properties, and chemical composition, of the modified biphasic solvent systems.

Thus, the main aim of this work was to test thermodynamic tools and find rational procedures to support the design of these chromatographic processes, to purify and fractionate phenolic compounds present in natural matrices, while reducing the experimental efforts, especially in the preliminary stages.

An in-depth literature review on the solubility and partition coefficients data was carried for the selected systems. Complementing the information, the partition coefficients of the four solutes in four binary biphasic systems (1-octanol/water, ethyl acetate/water, 1-butanol/water, and heptane/methanol), and in two quaternary systems (mixture $\mathrm{N}$ of the Arizona system composed of equal volumes of the four solvents, both in the original and green versions) were experimentally measured at $298.2 \mathrm{~K}$. The 1-octanol/water partition coefficient was measured as it is a well-known parameter for the characterization of the lipophilicity of a given solute. The other binary solvent systems are representatives of the polarity extremes of the Arizona system and the ethyl acetate/butanol/water ternary system. Mixture $\mathrm{N}$ is the middle biphasic system of the Arizona system.

Finally, the fully predictive Conductor-like Screening Model for Real Solvents (COSMO-RS) model [20], and the semi-predictive Non-random Two-Liquid Segment Activity Coefficient (NRTL-SAC) model [21] were applied to the description of the partition coefficients and their abilities to represent the experimental data discussed.
Table 1

Mass purity (\%), CAS number, and source of the organic compounds used in this work.

\begin{tabular}{llll}
\hline Compound & Mass purity (\%) & CAS number & Source \\
\hline trans-Ferulic acid & $\geq 99.9$ & $537-73-5$ & Alfa Aesar \\
(S)-Hesperetin & $\geq 98.0$ & $520-33-2$ & Cayman Chemicals \\
Quercetin & $\geq 95.0$ & $117-39-5$ & Sigma-Aldrich \\
Vanillin & $\geq 99.0$ & $121-33-5$ & Sigma-Aldrich \\
Methanol & $\geq 99.9$ & $67-56-1$ & J. T. Baker \\
Ethanol & $\geq 99.8$ & $64-17-5$ & Fisher \\
1-Butanol & $\geq 99,5$ & $71-36-3$ & Sigma-Aldrich \\
1-Octanol & $\geq 99.0$ & $111-87-5$ & Carlo Erba \\
Ethyl acetate & $\geq 99.9$ & $141-78-6$ & Carlo Erba \\
Heptane & $\geq 99.0$ & $142-82-5$ & Honeywell \\
$(R)-(+)-L i m o n e n e$ & $\geq 97.0$ & $5989-27-5$ & Sigma-Aldrich
\end{tabular}

a The purity was obtained in the certificate of analysis issued by the manufacturer.

\section{Material and methods}

\subsection{Chemicals}

The solutes and organic solvents were used as received from the supplier and stored at room temperature. To avoid water contamination, all the solids were kept in desiccators. The mass purity and source of the organic compounds are listed in Table 1. The water used was ultrapure quality (resistivity of $18.2 \mathrm{M} \Omega \bullet \mathrm{cm}$, free particles $<0.22 \mu \mathrm{m}$ and total organic carbon $\left.<5 \mu \mathrm{g} \bullet \mathrm{dm}^{-3}\right)$.

\subsection{Partition coefficient experiments}

The partition coefficients were measured by the conventional analytical shake-flask method, which has been extensively applied to assess the distribution of biomolecules in biphasic systems $[22,23]$ and provides accurate results for moderate $\log (K)$ values (typically in the range between -3 and 3) [24,25]. First, a sample of around $7 \mathrm{mg}$ (model ABT 100-5 M, Kern, $\pm 0.01 \mathrm{mg}$ ) of the solute was dissolved in one of the solvents of the biphasic system, ensuring that its maximum concentration in each phase was lower than $0.01 \mathrm{~mol} / \mathrm{L}$ in all the studied systems [25].

Then, the solution was transferred to a graduated glass tube along with predefined volumetric proportions of the other solvents, resulting in a total volume of $14 \mathrm{ml}$. The tubes were manually inverted at room temperature for five minutes (around 100 times), as recommended by Leo et al. [26], before being placed in a thermostatic equipment (Eppendorf ThermoMixer C) under continuous agitation $(300 \mathrm{rpm})$ at $(298.2 \pm 0.5) \mathrm{K}$ for at least $5 \mathrm{~h}$. Finally, the tubes were allowed to rest overnight (at least $15 \mathrm{~h}$ ) to ensure complete phase separation. All the shake-flask experiments were prepared in duplicate.

As recommended by the OECD [25], in the case of the octanolwater binary systems, the solvents were pre-saturated before implementing the procedure above to avoid the micelle formation. Then to ensure the complete phase separation, the tubes were centrifuged at $1500 \mathrm{rpm}$ and $298 \mathrm{~K}$ for $40 \mathrm{~min}$ (5800 series, Eppendorf).

The concentration of the solutes in each phase was determined by UV-Vis spectroscopy (model V-730, Jasco). An adequate volume was collected from each phase using all-glass syringes and diluted in either pure ethanol or a mixture of ethanol-water (wt\% 50:50). For each solute, calibration curves were built using at least seven standard solutions $\left(\mathrm{R}^{2}>0.999\right)$ at the wavelength of maximum absorbance (Table S1 of Supporting Information). At least two independent samples from each phase were analyzed. 


\section{Theory}

\subsection{The partition coefficients}

The partition coefficient of a nonelectrolyte organic solute in a biphasic solvent system $\left(P_{I}\right)$ is related to the activity coefficients at infinite dilution $\gamma_{I}{ }^{\infty}$ of species $I$ by the following expression derived from equilibrium thermodynamics [27,28]:

$P_{I}=\frac{x_{I}^{u}}{x_{I}^{l}}=\frac{\gamma_{I}^{l, \infty}}{\gamma_{I}^{u, \infty},} ; x_{I} \rightarrow 0$

where the superscripts $u$ and $l$ stand for the immiscible upper and lower phases, respectively, and $x_{I}$ is the mole fraction of the same compound $I$. In this work, the activity coefficients at infinite dilution were estimated by the COSMO-RS and NRTL-SAC models. Since the partition coefficients are typically reported as the ratio of molar concentrations, the values calculated though Eq. (1) can be converted using the approximate relation:

$K_{I}=\frac{c_{I}{ }^{u}}{c_{I}{ }^{l}}=P_{I} \cdot \frac{\sum_{j=1}^{n} x_{j}^{l} v_{j}}{\sum_{j=1}^{n} x_{j}{ }^{u} v_{j}}$

where $c$ corresponds to the molar concentration, $v_{j}$ is the molar volume of pure component $j$, and $n$ is the number of compounds.

For the calculation of the partition coefficients, the phase compositions of each biphasic system are required, and the data used in this work are shown in Table S2 of SI [29-31].

\subsection{The COSMO-RS model}

The COSMO-RS model (Conductor like Screening Model for Real Solvents) is a fully predictive model based on quantum chemistry that calculates the chemical potential of all components in a liquid mixture only through $a b$ initio calculations [20,32,33]. The activity coefficients of the solutes in the different mixtures were estimated based on the chemical potential, and the partition of a solute between the two liquid phases was calculated using Eq. (1) and (2). The details for COSMO-RS calculations can be found in our previous work [34]. In brief, prior to the COSMO-RS calculation, the geometry of the solute and solvents were optimized using TURBOMOLE V7.3 2018 software program package using density functional theory level and utilizing the BP functional B88-p86 with a triple- $\zeta$ valence polarized basis set (TZVP) and the resolution of identity standard (RI) approximation. Then, the activity coefficient of the solute in the liquid mixture was determined using COSMOthermX software and by applying the parameterization BP_TZVP_C30_1801 [35].

\subsection{The NRTL-SAC model}

The NRTL-SAC model, proposed by Chen and Song [21], has been widely employed to describe solubilities of biomolecules and pharmaceutical ingredients in pure and mixed solvents [21,36-49], and much less frequently to describe partition coefficients [28,5052]. The model describes the activity coefficient of a solute $I\left(\gamma_{I}\right)$ in a solution as the sum of two contributions:

$\ln \gamma_{I}=\ln \gamma_{I}^{C}+\ln \gamma_{I}^{R}$

where $\gamma_{I}^{C}$ and $\gamma_{I}^{R}$ represent the combinatorial and the residual terms of the activity coefficient of the solute, respectively. The complete set of equations for the calculation of these parameters are given elsewhere $[21,48]$. Briefly, the model proposes four conceptual segment descriptors to describe each molecule: $X$ (hydrophobicity), $Y$ - (polar repulsive), $Y+$ (polar attractive) and $Z$ (hydrophilicity). These parameters have already been reported for all the solvents studied in this work, with the exception of limonene,
[21,48,49] and the solutes ferulic acid [47], quercetin [49] and hesperetin [37].

The activity coefficients obtained by the NRTL-SAC model were not only used to describe the partition coefficients, but also the solubility of the solutes in different solvents, in order to have a more robust dataset to estimate the missing parameters. According to classical thermodynamics, giving a set of reasonable assumptions, the solubility of a solid solute in a liquid mixture can be calculated by [53]:

$\ln x_{I}=\frac{\Delta_{m} H}{R T_{m}}\left(1-\frac{T_{m}}{T}\right)-\frac{\Delta C_{p}}{R}\left(1+\ln \frac{T_{m}}{T}-\frac{T_{m}}{T}\right)-\ln \gamma_{I}$

where $\Delta_{m} H$ and $T_{m}$ are the melting enthalpy and temperature of the solute, respectively; $R$ is the ideal gas constant; and $\Delta C_{p}$ is the difference between the molar heat capacity of the solute in the liquid and solid phases. This heat capacity term is often negligible [54] and Eq. (4) can be reduced to:

$\ln x_{I}=\frac{\Delta_{m} H}{R T_{m}}\left(1-\frac{T_{m}}{T}\right)-\ln \gamma_{I}$

The melting temperatures and enthalpies of the solutes used in this work were collected from the open literature [47,49,55-57], and are listed in Table S3 of SI.

\section{Results and discussion}

\subsection{Experimental partition coefficients}

The experimental partition coefficients of ferulic acid, (S)hesperetin, quercetin, and vanillin in 4 binary biphasic systems (heptane/methanol, 1-butanol/water, 1-octanol/water, and ethyl acetate/water), and in the two quaternary biphasic systems Arizona N (water/methanol/ethyl acetate/heptane, in the proportion 1/1/1/1) and green Arizona $N$ (water/ethanol/ethyl acetate/limonene, in the proportion $1 / 1 / 1 / 1$ ) are presented in Table 2 , together with experimental literature data obtained at $298.2 \pm 3 \mathrm{~K}$. In some cases, the experiments were reported at room temperature, but were included here for comparison purposes.

As mentioned before, each $\log (K)$ measured in this work is the average of two independent shake-flask measurements. The overall consistency of the measurements is checked by the low standard deviations observed in Table 2, with $2.8 \%$ average for the coefficient of variation. In addition, the total amount of solid placed in the tubes was compared to the values calculated from the material balance, using the measured concentrations in each phase, resulting in global average relative deviations of $4.3 \%$.

The partition coefficients obtained in this work present absolute deviations (in log units) from the average literature value of lower than 0.3 (a reference value taken from [25]), with the exception of quercetin in methanol/heptane and 1-butanol/water biphasic systems. In the octanol/water system, the $\log (K)$ values from the literature are more scattered, presenting a standard deviation higher than 0.3 for ferulic acid, (S)-hesperetin and quercetin. In particular, the octanol/water partition coefficient for quercetin reported by Marlot [58] is considerably lower than the other data available in the open literature, which is not expected due to the hydrophobic nature of quercetin [70,71]. It is worth mentioning that a pre-saturation step of the phases, preferably through continuous stirring [72], is highly recommended prior to the determination of octanol-water partition coefficients through the shake-flask method $[22,25,72]$. Tests performed with $(S)$-hesperetin showed that this pre-saturation step was not necessary for the other binary solvent systems (deviations in $\log (K)$ of \pm 0.01 ).

To the best of our knowledge, the $\log (K)$ of all the studied solutes in the green Arizona $\mathrm{N}$ system are reported here for the first time. In general, replacing methanol and heptane by ethanol and 
Table 2

Experimental $\log (K)$ values obtained in this work and found in the literature for the selected biphasic systems.

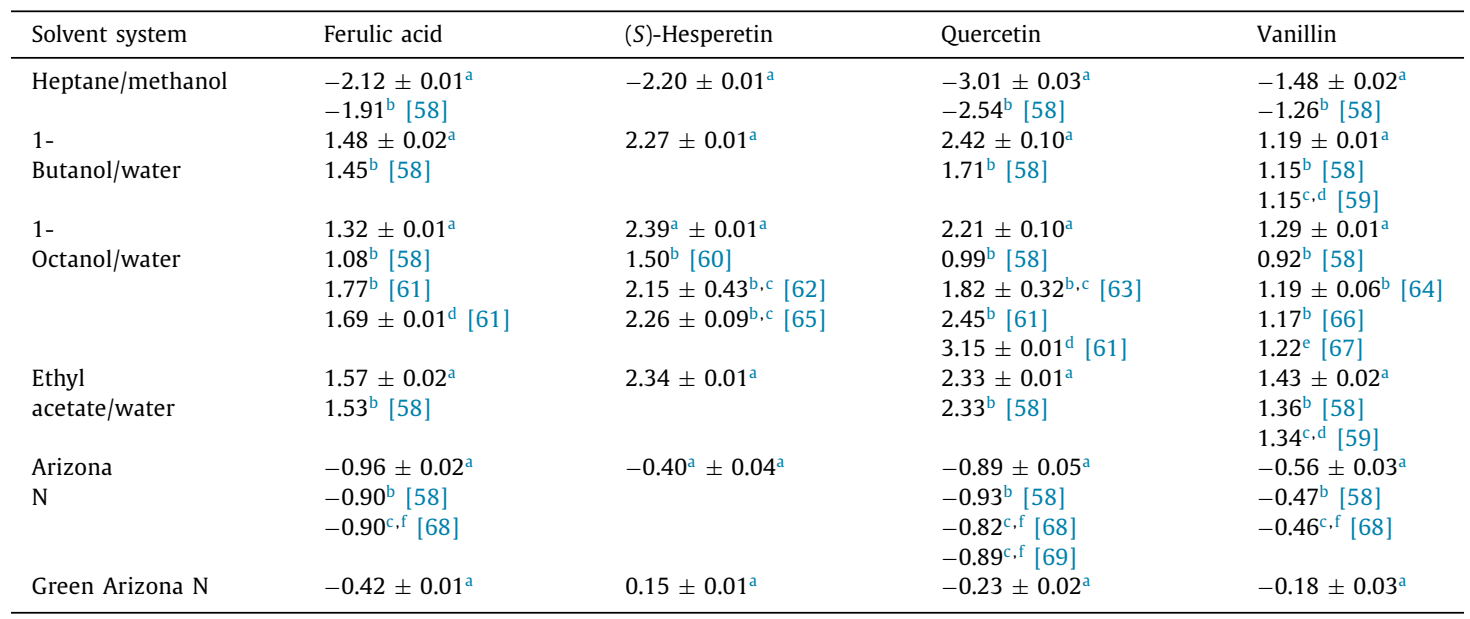

a Measured in this work. Temperature and pressure standard uncertainties are $u(T)=0.50 \mathrm{~K}$ and $u^{r}(p)=0.05$, respectively. Standard deviations are placed after the minus-plus sign.

b Obtained by the shake-flask method.

c Measured at room temperature.

d Experimentally determined by reversed phase HPLC analysis.

e Experimentally measured by a slow equilibrium method through an octanol drop apparatus.

${ }^{\mathrm{f}}$ Obtained by countercurrent chromatography (CCC).

limonene, respectively, increases the $\log (K)$ values of the phenolic compounds between 0.3 (vanillin) and 0.7 (quercetin) log units. In fact, the substitution of methanol by ethanol increases the alcohol content in the upper phase, and since the phenolic compounds are typically soluble in alcohols, an increase in the partition coefficient is expected. The presence of limonene instead of heptane also increases the overall polarity of the green Arizona in comparison with the original system [19], leading to higher $\log (K)$.

The observed $\log (K)$ of the solutes in the studied organic/aqueous biphasic binary systems are higher than 1 , indicating that the studied phenolic compounds present a more lipophilic character, particularly for quercetin and $(S)$-hesperetin $(\log (K)>$ 2 ). On the other hand, the negative $\log (K)$ obtained for the heptane/methanol systems reflects the poor affinity of the phenolic compounds towards hydrocarbon organic solvents. For the original and green versions of the Arizona $\mathrm{N}$ system, the obtained $\log (K)$ values are negative, except for $(S)$-hesperetin in the green Arizona $\mathrm{N}$ system. For an optimal CCC separation, $\log (K)$ should vary between -0.4 and 0.4 [15]. Our results show that the green version (of intermediate polarity) is an adequate solvent system for the separation of the studied phenolic compounds. In the case of the Arizona family, a more polar solvent system between A (ethyl acetate/water) and $\mathrm{N}$ should be selected.

\subsection{Thermodynamic modeling}

\subsubsection{COSMO-RS model}

The partition coefficients predicted with the COSMO-RS model are compared with the experimental values measured in this work in Figure S1. To quantify the deviations, the root-mean square deviations (RMSD) were calculated for each solute as follows:

RMSD $=\sqrt{\left[\frac{\sum_{i}\left(\log K_{i}^{\text {exp }}-\log K_{i}^{\text {calc }}\right)^{2}}{n}\right]}$

where superscripts "exp" and "calc" mean the experimental and calculated values, respectively, $n$ is the total number of data points and $i$ covers all the solvent systems. As can be seen, RMSD from the experimental data measured in this work for vanillin, ferulic acid, quercetin and (S)-hesperetin were respectively of $0.48,0.78$, 1.06 and 1.52 (6 data points for each solute), respectively.

The model seems to overestimate the hydrophobicity of the studied molecules, by over predicting $\log (K)$ in the binary organic/aqueous biphasic systems, more significantly in the ethyl acetate/water biphasic system. Furthermore, in the quaternary solvent mixtures (system Arizona $\mathrm{N}$ and green Arizona $\mathrm{N}$ ), the model erroneously predicts that the solutes will preferably partition to the upper phase, though it is able to qualitatively predict the change in $\log (K)$ when moving from the classical to the green version of the Arizona systems for all solutes.

To achieve a more comprehensive overview of the quality of the predictions, a literature review on the partition coefficients of the selected solutes in biphasic systems composed of water and organic solvents was carried out, aiming to cover a wide polarity range as in the case of the Arizona and HEMWat solvent systems. A comparison between the experimental data (collected in the literature and measured in this work) and COSMO-RS results is summarized in Fig. 1. The detailed information can be found in Table S4 of SI.

Considering all the available data points, the average RMSD obtained for the systems containing the flavonoid aglycones, $(S)$ hesperetin ( 6 data points measured in this work) and quercetin (16 data points), were 1.52 and 1.74 , respectively. Lower RMSD were obtained for the partition coefficients of the less structurally complex ferulic acid (RSMD $=0.96,13$ data points) and vanillin molecules (RSMD $=0.57,26$ data points). Considering the additional available data points for quercetin, ferulic acid, and vanillin, the average RMSD increased when compared to the values obtained using only the data measured in this work. Values of RMSD lower than 0.5 have been considered acceptable $[23,73,74]$, but were not consistently obtained in this work with COSMO-RS.

\subsubsection{NRTL-SAC model}

In previous works, Ren and co-workers [28,50-52] proposed a procedure to optimize the NRTL-SAC solute descriptors for a given solvent system family (e.g., HEMWat, Arizona) based on the fitting of a minimal set of experimental partition coefficient data. The authors suggested the inclusion of $\log (K)$ data measured for the same solvent family but with different global composi- 
Table 3

The NRTL-SAC molecular parameters and RMSD obtained in the correlation.

\begin{tabular}{lllllcr}
\hline Compound & $X$ & $Y-$ & $Y+$ & $Z$ & RMSD in $\log (\boldsymbol{x})$ & RMSD in $\log (K)$ \\
\hline Ferulic acid & 0.530 & 0.000 & 0.793 & 1.029 & 0.48 & 0.24 \\
(S)-Hesperetin & 0.769 & 0.000 & 0.627 & 1.211 & 0.36 & 0.07 \\
Quercetin & 0.798 & 0.000 & 0.874 & 1.633 & 0.06 \\
Vanillin & 0.456 & 0.000 & 0.631 & 0.662 & 0.10 \\
\hline
\end{tabular}

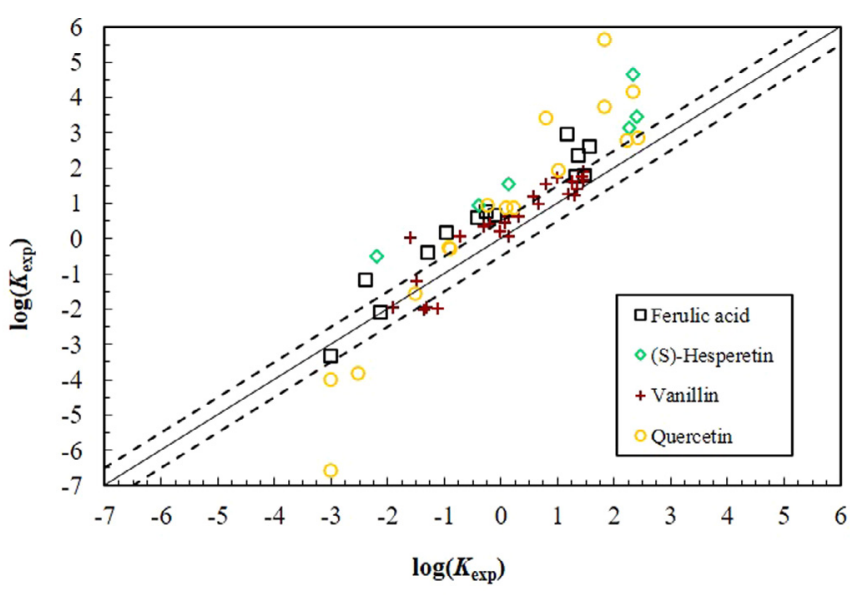

Fig. 1. Comparison between the experimental (collected in the literature and measured in this work) and the predicted partition coefficients using the COSMO-RS model. The dashed lines correspond to $\log (K) \pm 0.5$.

tions (e.g., HEMWat $1 / 9 / 1 / 9,1 / 1 / 1 / 1,9 / 1 / 9 / 1$ ) in the correlation set. In this work, an alternative approach is suggested as only $\log (K)$ obtained in the binary solvent systems measured in this work (heptane/methanol, 1-butanol/water, 1-octanol/water, ethyl acetate/water) were included in the correlation set in combination with solubility data in pure solvents (44 data points in total) available in the literature $[37,47,75-81]$.

The optimization procedure was carried out using the lsqnonlin function (MATLAB R2020b) based on the nonlinear least-squares curve fitting analysis and the following objective function $(F)$ :

$F=\sum_{i}\left(a_{i}{ }^{\text {exp }}-a_{i}^{\text {calc }}\right)^{2}$

where $a_{i}$ represents the properties used in the correlation set ( $x_{i}$ and $K_{i}$ ) for the solvent system $i$ and the superscripts "exp" and "calc" mean the experimental and calculated values, respectively. The NRTL-SAC segment descriptors and the RMSD values obtained in the correlation step are presented in Table 3. The molecular parameters of the solvents were already available in the literature, except for limonene. For that compound, the parameters were estimated using partition data from literature of limonene in 1octanol/water [82] and hexane/acetonitrile [83] biphasic systems, with RMSD of 0.27 (Table S6). As in the case of other hydrophobic solvents (alkanes and aromatics) [48], limonene was represented only by the hydrophobic segment $(X=0.965)$, as reported in Table S5.

The NRTL-SAC segment descriptors obtained in this work are considerably different from the values reported in previous works for ferulic acid [47], hesperetin [37] and quercetin [49], which were fitted using mostly solubility data in water and in pure organic polar solvents. From our experience [36,45,47], the robustness of the model descriptors is associated with the chemical diversity of solvent systems used in the correlation step, and differences in the fitted parameters were expected since partition coefficients in less polar biphasic solvent systems (e.g., heptane/methanol) were included in the data set. As defined by Chen and Song [21], seg- ment $Z$ represents molecular surfaces capable of hydrogen bonding and $X$ accounts for the hydrophobic contribution, which are expected contributions in the studied solutes. All the other surface interactions are lumped in the polar segments $(Y$ - and $Y+)$ [38].

As shown in Table 3, some trends can be identified in the NRTLSAC segment parameters of the solutes. The hydrophilic segment $Z$ increases in the order: vanillin $<$ ferulic acid $<(S)$-hesperetin $<$ quercetin, which is consistent with the increase of the total number of hydrogen bond donor and acceptor sites available in the molecules (presented in Table S7 of SI). The hydrophobic segment $X$ is associated with the molecular surface area averse to hydrogen bonding. As can be seen, $X$ increases with the number of carbon atoms of the solutes, except when comparing the values of $(S)$ hesperetin $(X=0.769 ; 16$ carbons $)$ and quercetin $(X=0.798 ; 15$ carbons), which are quite similar. Regarding the polar segments, the fitted parameters suggest that no significant polar attractive $(Y-=0)$ and moderate polar repulsive $(0<Y+<1)$ regions are observed in the molecular surfaces of the solutes.

In Fig. 2, the big picture of the correlation step is shown, where partition coefficients (a) and solubilities (b) calculated by the NRTL-SAC are presented as function of the experimental data. The values used in the construction of Fig. 2 are listed in Tables S6 and $\mathrm{S} 8$ of the SI.

Satisfactory correlation results (RMSD $\leq 0.5)$ were obtained in $\log (K)$, presenting maximum RMSD of 0.10 (vanillin). The RMSD values obtained in the correlation of the partition coefficients of binary solvent systems are within the same order of magnitude of the RMSD found by Ren et al. [28,50] using $\log (K)$ of quaternary solvent systems. Larger deviations are observed in the correlation of the solubility data, in particular for ferulic acid (RMSD $=0.48$, 20 data points) and quercetin (RMSD $=0.36,10$ data points). As discussed in previous works [36,47], the quality of the solubility calculations is associated with the accuracy of the melting properties of the solutes, which are unknown for some phenolic compounds due to their thermal decomposition before melting [84,85].

To test the predictive capability of the model, the partition coefficients in other binary and quaternary solvent systems (45 data points) measured in this work and found in literature [58,59,68,69,86-98], were calculated and the prediction results are shown in Fig. 3 and summarized in Table S9.

In general, the NRTL-SAC model provides better predictions than COSMO-RS, presenting RMSD of 0.28 for ferulic acid (9 data points), 0.02 for ( $S$ )-hesperetin ( 2 data points), 0.73 for quercetin (12 data points) and 0.20 for vanillin (22 data points). Most of the predicted values (87\%) are distributed within the $\log (K) \pm 0.5$ regions demarked in Fig. 3, with a few outliers for quercetin (heptane/acetonitrile, methyl-tert-butyl ether/water, 2-butanone/water, Arizona $\mathrm{Q}$ and Arizona $\mathrm{Y}$ ) and another for ferulic acid (Arizona Y). The volumetric proportions of heptane, ethyl acetate, methanol and water in the Arizona $Q$ and $Y$ systems are 3:2:3:2 and 19:1:19:1, respectively. For vanillin, the NRTL-SAC predictions are in excellent agreement with the experimental data for a large variety of solvent systems, including some which are structurally different from those used in the correlation step, such as dichloromethane/water, toluene/water and methyl isobutyl ketone/water. 


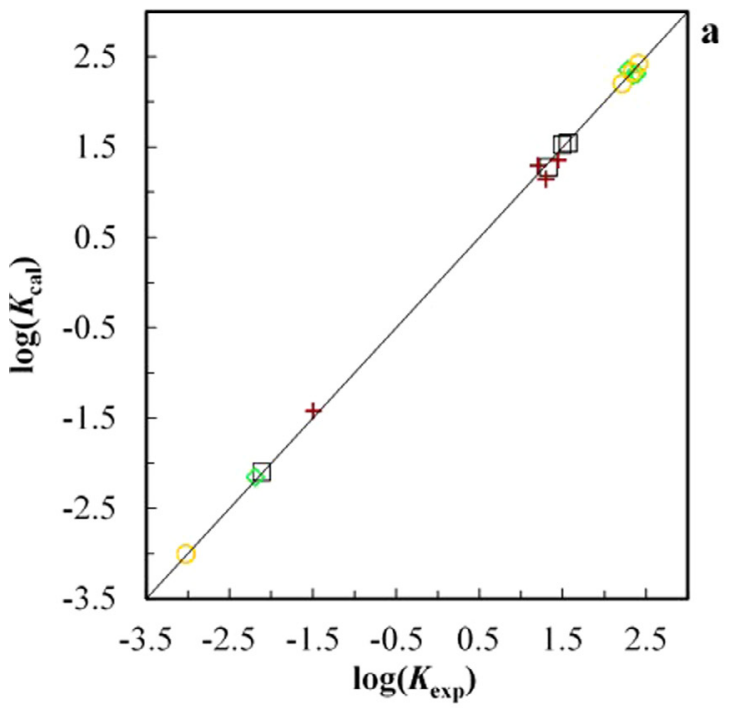

$\square$ Ferulic acid $\diamond(\mathrm{S})$-Hesperetin + Vanillin Quercetin

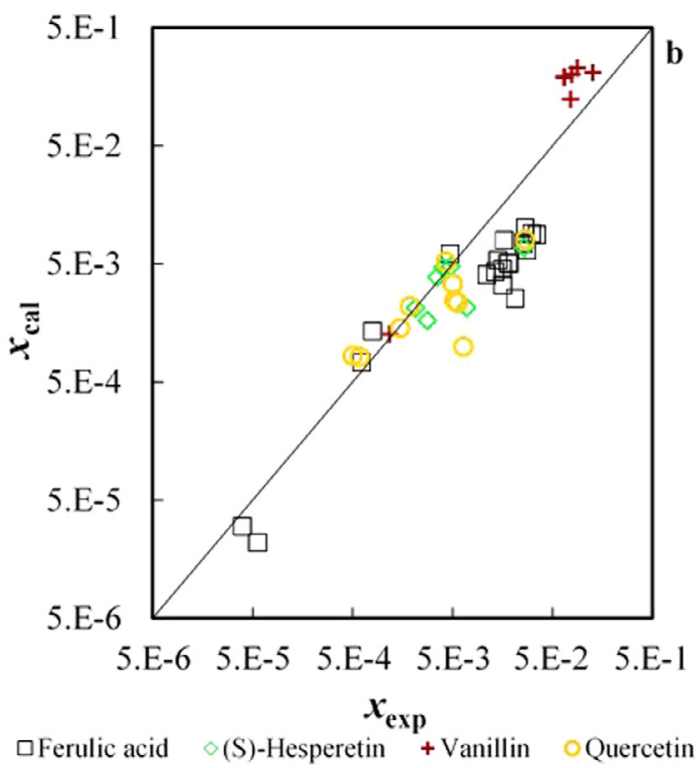

Fig. 2. Comparison between the experimental and correlated data using the NRTL-SAC model: a) partition coefficients; b) solubilities.

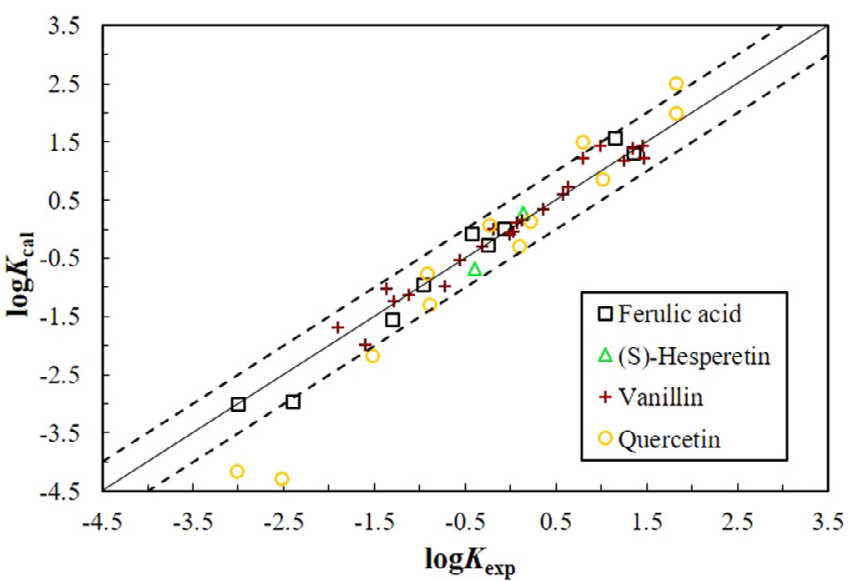

Fig. 3. Comparison between the experimental and the predicted partition coefficients using the NRTL-SAC model. The dashed lines correspond to $\log (K) \pm 0.5$.

As discussed before, the search for an appropriate solvent system for a specific CCC separation is a laborious task [52], and the use of computational tools for narrowing the number of experiments is welcome. To illustrate the predictive capability of the NRTL-SAC and COSMO-RS models, the $\log (K)$ of the studied phenolic compounds in the conventional and green versions of the Arizona solvent system are presented in Fig. 4 and Figure S2, respectively.

This family of solvents was selected as an example because it covers a large polarity range from the most polar ethyl acetate/water biphasic system (system A) to the least polar heptane/methanol (system Z) and it is widely applied in countercurrent chromatographic techniques. As expected, both models can capture the decrease in $\log (K)$, though much larger deviations are obtained with the COSMO-RS model, as shown and discussed before. Better results are obtained with the NRTL-SAC model, particularly in the solvent systems richer in water, underpredicting the values in the more apolar systems. This situation is more evident for quercetin.

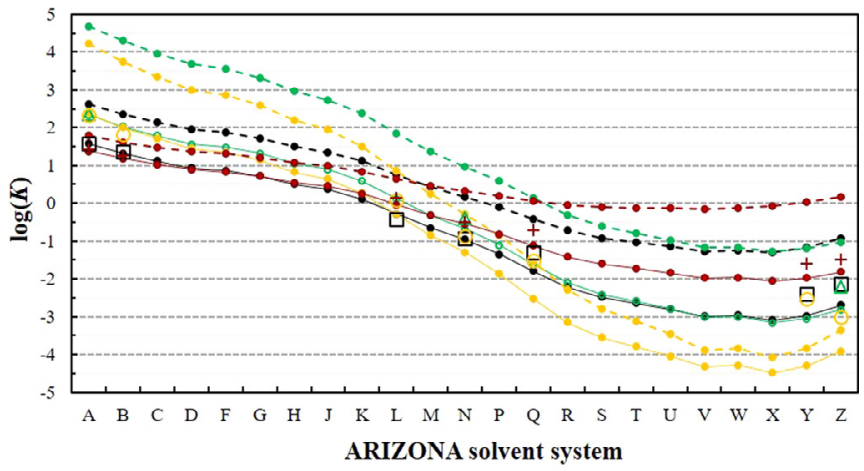

Fig. 4. $\log (K)$ values as a function of the conventional Arizona solvent systems: open symbols - experimental data; filled circles connected by solid lines - NRTL-SAC model; filled circles connected by dashed lines - COSMO-RS model. Color codes: black - ferulic acid; green - hesperetin; red - vanillin; yellow - quercetin. Lines are guides for the eyes.

\section{Conclusions}

The experimental partition coefficients of ferulic acid, $(S)$ hesperetin, quercetin, and vanillin were measured, at $298 \mathrm{~K}$, in six biphasic solvent systems. To our knowledge, the data obtained in the green Arizona $\mathrm{N}$ system composed of water/ethyl acetate/ethanol/limonene and for (S)-hesperetin (flavanone) in five biphasic systems are reported for the first time. The remaining data measured in this work present absolute deviations (in $\log$ units) from the average literature value lower than 0.3 , with some exceptions. Higher deviations are obtained for the water/1-octanol system and/or when $\log (K)>2$, stressing the importance of reporting and evaluating this scarce experimental information, which is essential for developing modeling tools. These experimental data were added to the literature database comprising 61 data points in total.

The COSMO-RS model resulted in global RMSD in $\log (K)$ of 1.17 , which can be considered acceptable given its fully predictive character. In general, it is able to describe the partition coefficient differences between the biphasic systems qualitatively. Better results 
are found for the solutes with lower molecular weight, vanillin and ferulic acid, for which RMSD of 0.57 and 0.96 were obtained, respectively. Improving considerably the description of the experimental data, the semi-empirical NRTL-SAC model was applied. Global RMSDs values of 0.07 and 0.41 were obtained for the correlated $\log (K)$ and solubility data, respectively, and a global RMSD of 0.43 for the predictions in $\log (K)$.

The results suggest applying the COSMO-RS model for preliminary solvent screening, mainly when no experimental information is available for the solvents and/or solutes under study, such as in the case of limonene or other less conventional green solvents. If a few experimental data sets (solubility, partition, etc.) are available in a diverse group of solvents, a more quantitative description of the partition coefficients can be achieved using the NRTL-SAC model. It can generate very good estimates of the partition coefficients change in a series of systems, as demonstrated here with the Arizona family, being a significant contribution when selecting biphasic liquid systems for partition chromatography.

\section{Declaration of Competing Interest}

The authors declare that they have no known competing financial interests or personal relationships that could have appeared to influence the work reported in this paper.

\section{CRediT authorship contribution statement}

Sérgio M. Vilas-Boas: Investigation, Methodology, Software, Data curation, Writing - original draft. Isabella W. Cordova: Investigation, Validation. Kiki A. Kurnia: Investigation, Software. Heloísa H.S. Almeida: Investigation, Methodology. Priscilla S. Gaschi: Supervision. João A.P. Coutinho: Conceptualization, Writing - review \& editing, Supervision, Funding acquisition. Simão P. Pinho: Conceptualization, Writing - review \& editing, Supervision, Project administration. Olga Ferreira: Conceptualization, Writing review \& editing, Supervision, Project administration, Funding acquisition.

\section{Acknowledgements}

This work was developed within the scope of the project AllNat - POCI-01-0145-FEDER-030463 (PTDC/EQU-EPQ/30463/2017), funded by FEDER funds through COMPETE2020 - Prog. Operacional Competitividade e Internacionalização (POCI), and by national funds through the Foundation for Science and Technology (FCT/MCTES). Support was also provided by CIMO-Mountain Research Center, UIDB/00690/2020 and CICECO-Aveiro Institute of Materials, UIDB/50011/ 2020 \& UIDP/50011/2020, both financed by national funds through FCT/MCTES. S. M. Vilas-Boas thanks FCT and the European Social Fund (ESF) for his Ph.D. grant (SFRH/BD/138149/2018). I. W. Cordova and H. Almeida also thank project AllNat - POCI-01-0145-FEDER-030463 for their contracts.

\section{Supplementary materials}

Supplementary material associated with this article can be found, in the online version, at doi:10.1016/j.chroma.2022.462859.

\section{References}

[1] R.N. Cavalcanti, T. Forster-Carneiro, M.T.M.S. Gomes, M.A. Rostagno, J.M. Prado, M.A.A. Meireles, Uses and applications of extracts from natural sources, in: M.A. Rostagno, M.A. Rostagno (Eds.), Natural Product Extraction: Principles, The Royal Chemistry of Society, 2013: pp. 1-57.

[2] W. Xiao, F. Lei, Z. Hengqiang, L. Xiaojing, Isolation and purification of natural products, in: M.A. Rostagno, J.M. Prado (Eds.), Natural Product Extraction: Principles, Royal Society of Chemistry, 2013: pp. 314-362.
[3] C.F. Poole, Milestones in the Development of Liquid-Phase Extraction techniques, in: Liquid-Phase Extraction, Elsevier, 2020: pp. 1-44.

[4] A. Marston, K. Hostettmann, Developments in the application of counter-current chromatography to plant analysis, J. Chromatogr. A. 1112 (2006) 181 194.

[5] L. Lorántfy, R. Örkenyi, D. Rutterschmid, D. Bakonyi, J. Faragó, G. Dargó, Á. Könczöl, Continuous industrial-scale centrifugal partition chromatography with automatic solvent system handling: concept and instrumentation, Org. Process Res. Dev. 24 (2020) 2676-2688.

[6] Y. Liu, J.B. Friesen, J.B. McAlpine, G.F. Pauli, Solvent system selection strategies in countercurrent separation, Planta Med. 81 (2015) 1582-1591.

[7] D.P. Ward, M. Cárdenas-Fernández, P. Hewitson, S. Ignatova, G.J. Lye, Centrifugal partition chromatography in a biorefinery context: separation of monosaccharides from hydrolysed sugar beet pulp, J. Chromatogr. A. 1411 (2015) 84-91.

[8] J.H.P.M. Santos, M.R. Almeida, C.I.R. Martins, A.C.R.V. Dias, M.G. Freire, J.A.P. Coutinho, S.P.M. Ventura, Separation of phenolic compounds by centrifugal partition chromatography, Green Chem 20 (2018) 1906-1916.

[9] A. Dubuis, A. Le Masle, L. Chahen, E. Destandau, N. Charon, Centrifugal partition chromatography as a fractionation tool for the analysis of lignocellulosic biomass products by liquid chromatography coupled to mass spectrometry, J. Chromatogr. A. 1597 (2019) 159-166.

[10] E. Hopmann, A. Frey, M. Minceva, A priori selection of the mobile and stationary phase in centrifugal partition chromatography and counter-current chromatography, J. Chromatogr. A. 1238 (2012) 68-76.

[11] Y. Liu, P. Kuang, S. Guo, Q. Sun, T. Xue, H. Li, An overview of recent progress in solvent systems, additives and modifiers of counter current chromatography, New J. Chem. 42 (2018) 6584-6600.

[12] Y. Ito, Golden rules and pitfalls in selecting optimum conditions for high-speed counter-current chromatography, J. Chromatogr. A. 1065 (2005) 145-168.

[13] I.J. Garrard, Simple approach to the development of a CCC solvent selection protocol suitable for automation, J. Liq. Chromatogr. Relat. Technol. 28 (2005) 1923-1935.

[14] A.P. Foucault, Centrifugal Partition Chromatography, Marcel Dekker, 1994.

[15] J.B. Friesen, G.F. Pauli, G.U.E.S.S. - A generally useful estimate of solvent systems in CCC, J. Liq. Chromatogr. Relat. Technol. 28 (2005) 2777-2806.

[16] K. Skalicka-Woźniak, I. Garrard, A comprehensive classification of solvent systems used for natural product purifications in countercurrent and centrifugal partition chromatography, Nat. Prod. Rep. 32 (2015) 1556-1561.

[17] F. Das Neves Costa, G.G. Leitão, Strategies of solvent system selection for the isolation of flavonoids by countercurrent chromatography, J. Sep. Sci. 33 (2010) 336-347.

[18] K. Faure, E. Bouju, P. Suchet, A. Berthod, Limonene in Arizona liquid systems used in countercurrent chromatography. I Physicochemical properties, Anal. Bioanal. Chem. 406 (2014) 5909-5917.

[19] K. Faure, E. Bouju, J. Doby, A. Berthod, Limonene in Arizona liquid systems used in countercurrent chromatography. II Polarity and stationary-phase retention, Anal. Bioanal. Chem. 406 (2014) 5919-5926.

[20] A. Klamt, Conductor-like screening model for real solvents: a new approach to the quantitative calculation of solvation phenomena, J. Phys. Chem. 99 (1995) 2224-2235.

[21] C.-C. Chen, Y. Song, Solubility modeling with NRTL segment activity coefficient model, Ind. Eng. Chem. Res. 43 (2004) 8354-8362.

[22] J. Sangster, Octanol water partition coefficients of simple organic compounds, J. Phys. Chem. Ref. Data. 18 (1989) 1111-1229.

[23] L. Marlot, M. Batteau, K. Faure, Classification of biphasic solvent systems according to Abraham descriptors for countercurrent chromatography, J. Chromatogr. A. 1617 (2020) 460820.

[24] A. Berthod, S. Carda-Broch, Determination of liquid-liquid partition coefficients by separation methods, J. Chromatogr. A. 1037 (2004) 3-14.

[25] Organisation for Economic Co-operation and Development (OECD/OCDE), OECD GUIDELINE FOR THE TESTING OF CHEMICALS, Partition Coefficient (n-octanol/water): shake Flask Method, Oecd Giudeline Test. Chem. 107 (1995) $1-4$.

[26] A. Leo, C. Hansch, D. Elkins, Partition coefficients and their uses, Chem. Rev. 71 (1971) 525-616.

[27] E. Hopmann, W. Arlt, M. Minceva, Solvent system selection in counter-current chromatography using conductor-like screening model for real solvents, J. Chromatogr. A. 1218 (2011) 242-250.

[28] D.B. Ren, Z.H. Yang, Y.Z. Liang, Q. Ding, C. Chen, M.L. Ouyang, Correlation and prediction of partition coefficient using nonrandom two-liquid segment activity coefficient model for solvent system selection in counter-current chromatography separation, J. Chromatogr. A. 1301 (2013) 10-18.

[29] L.M. Casás, B. Orge, O. Ferreira, Liquid-liquid equilibria of mixtures containing methyl acetate + methanol + hexane or heptane, J. Chem. Eng. Data. 53 (2008) 89-93.

[30] M. Góral, B. Wiśniewska-Gocłowska, A. Mą̧zyński, Recommended liquid-liquid equilibrium data. Part 4. 1-alkanol-water systems, J. Phys. Chem. Ref. Data. 35 (2006) 1391-1414

[31] M. Góral, D.G. Shaw, A. McZyński, B. Wiśniewska-Gocowska, A. Jezierski, IUPAC-NIST solubility data series. 88. esters with water-revised and updated. Part 1. C2 to C4 esters, J. Phys. Chem. Ref. Data. 38 (2009) 1093-1127.

[32] A. Klamt, V. Jonas, T. Bürger, J.C.W. Lohrenz, Refinement and parametrization of COSMO-RS, J. Phys. Chem. A. 102 (1998) 5074-5085.

[33] A. Klamt, COSMO-RS: From quantum Chemistry to Fluid Phase Thermodynamics and Drug Design, 1st ed., Elsevier Science, 2005.

[34] K.A. Kurnia, S.P. Pinho, J.A.P. Coutinho, Evaluation of the conductor-like screen- 
ing model for real solvents for the prediction of the water activity coefficient at infinite dilution in ionic liquids, Ind. Eng. Chem. Res. 53 (2014) 12466-12475

[35] F. Eckert, A. Klamt, COSMOtherm, Version C30, Release 18 (2018)

[36] S.M. Vilas-Boas, V. Vieira, P. Brandão, R.S. Alves, J.A.P. Coutinho, S.P. Pinho, O. Ferreira, Solvent and temperature effects on the solubility of syringic, vanillic or veratric acids: experimental, modeling and solid phase studies, J. Mol. Liq. 289 (2019) 111089.

[37] O. Ferreira, S. Pinho, Solubility of flavonoids in pure solvents, Ind. Eng. Chem. Res. 51 (2012) 6586-6590.

[38] H.H. Tung, J. Tabora, N. Variankaval, D. Bakken, C.C. Chen, Prediction of pharmaceutical solubility via NRTL-SAC and COSMO-SAC, J. Pharm. Sci. 97 (2008) 1813-1820.

[39] P.B. Kokitar, E. Plocharczyk, C.-C. Chen, Modeling drug molecule solubility to identify optimal solvent systems for crystallization, Org. Process Res. Dev. 12 (2008) 249-256.

[40] F.L. Mota, A.P. Carneiro, A.J. Queimada, S.P. Pinho, E.A. Macedo, Temperature and solvent effects in the solubility of some pharmaceutical compounds: measurements and modeling, Eur. J. Pharm. Sci. 37 (2009) 499-507.

[41] F.L. Mota, A.J. Queimada, A.E. Andreatta, S.P. Pinho, E.A. Macedo, Calculation of drug-like molecules solubility using predictive activity coefficient models, Fluid Phase Equilib 322-323 (2012) 48-55.

[42] E. Sheikholeslamzadeh, S. Rohani, Solubility prediction of pharmaceutical and chemical compounds in pure and mixed solvents using predictive models, Ind. Eng. Chem. Res. 51 (2012) 464-473.

[43] W. Tang, Z. Wang, Y. Feng, C. Xie, J. Wang, C. Yang, J. Gong, Experimental determination and computational prediction of androstenedione solubility in alcohol + water mixtures, Ind. Eng. Chem. Res. 53 (2014) 11538-11549.

[44] E. Papadakis, A.K. Tula, R. Gani, Solvent selection methodology for pharmaceutical processes: solvent swap, Chem. Eng. Res. Des. 115 (2016) 443-461.

[45] S.M. Vilas-Boas, P. Brandão, M.A.R. Martins, L.P. Silva, T.B. Schreiner, L. Fernandes, O. Ferreira, S.P. Pinho, Solubility and solid phase studies of isomeric phenolic acids in pure solvents, J. Mol. Liq. 272 (2018) 1048-1057.

[46] M. Silva, J.C. García, M. Ottens, Polyphenol Liquid-Liquid Extraction Process Development Using NRTL-SAC, Ind. Eng. Chem. Res. 57 (2018) 9210-9221.

[47] S.M. Vilas-Boas, R.S. Alves, P. Brandão, L.M.A. Campos, J.A.P. Coutinho, S.P. Pinho, O. Ferreira, Solid-liquid phase equilibrium of trans-cinnamic acid, p-coumaric acid and ferulic acid in water and organic solvents: experimental and modelling studies, Fluid Phase Equilib 521 (2020) 112747.

[48] C.-C. Chen, P.A. Crafts, Correlation and prediction of drug molecule solubility with the NRTL-SAC model, Comput. Aided Chem. Eng. 21 (2006) 859-864.

[49] R. Aguda, C.-C. Chen, Solubility of nutraceutical compounds in generally recognized as safe solvents at 298K, Int. J. Chem. Eng. Appl. 7 (2016) 289294.

[50] D.B. Ren, L.Z. Yi, Y.H. Qin, Y.H. Yun, B.C. Deng, H.M. Lu, X.Q. Chen, Y.Z. Liang, Systematic and practical solvent system selection strategy based on the nonrandom two-liquid segment activity coefficient model for real-life counter-current chromatography separation, J. Chromatogr. A. 1393 (2015) 47-56.

[51] Y. Qin, Y. Liang, D. Ren, X. Qiu, X. Li, Separation of phenolic acids and flavonoids from Trollius chinensis Bunge by high speed counter-current chromatography, J. Chromatogr. B. 1001 (2015) 82-89.

[52] D. Ren, B. Han, Z. Xin, W. Liu, S. Ma, Y. Liang, L. Yi, Computation-aided separation of seven components from Spirodela polyrrhiza (L.) via counter-current chromatography, Sep. Purif. Technol. 165 (2016) 160-165.

[53] J.M. Prausnitz, R.N. Lichtenthaler, E.G. de Azevedo, Molecular Thermodynamics of Fluid-Phase Equilibria, Pretnice Hall PTR, 1999.

[54] M.A.R. Martins, S.P. Pinho, J.A.P. Coutinho, Insights into the nature of eutectic and deep eutectic mixtures, J. Solution Chem. 48 (2019) 962-982.

[55] L. Chebil, C. Humeau, J. Anthony, F. Dehez, J.M. Engasser, M. Ghoul, Solubility of flavonoids in organic solvents, J. Chem. Eng. Data. 52 (2007) 1552-1556.

[56] J. Singh, N.B. Singh, Phase equilibrium, crystallization behavior and thermodynamic studies of (m-dinitrobenzene + vanillin) eutectic system, J. Chem. Thermodyn. 89 (2015) 197-204.

[57] P. Gupta, T. Agrawal, S.S. Das, N.B. Singh, Phase equilibria and molecular interaction studies on (naphthols + vanillin) systems, J. Chem. Thermodyn. 48 (2012) 291-299.

[58] L. Marlot, Développement De Méthodes Bidimensionnelles Préparatives CPCxLC : Application à La Purification De Molécules D'intérêt Issues De Matrices Végétales, Université de Lyon, 2018.

[59] G. Lamprecht, K. Blochberger, Protocol for isolation of vanillin from ice cream and yoghurt to confirm the vanilla beans origin by 13C-EA-IRMS, Food Chem 114 (2009) 1130-1134.

[60] I.A. Suma, A. Kot-Wasik, A. Wasik, J. Namiésnik, C. Sârbu, Assessment of lipophilicity indices derived from retention behavior of antioxidant compounds in RP-HPLC, Molecules 22 (2017) 1-9.

[61] Z. Shi, J. He, T. Yao, W. Chang, RP-HPLC Determination of octanol-water partition coefficients for bioactive compounds from chinese herbal medicines, J. Liq. Chromatogr. Relat. Technol. 27 (2004) 465-479.

[62] Q.Q. Wang, J.-B. Shi, C. Chen, C. Huang, W.J. Tang, J. Li, Hesperetin derivatives: synthesis and anti-inflammatory activity, Bioorganic Med. Chem. Lett. 26 (2016) 1460-1465.

[63] J.A. Rothwell, A.J. Day, M.R.A. Morgan, Experimental determination of octanol-water partition coefficients of quercetin and related flavonoids, J. Agric. Food Chem. 53 (2005) 4355-4360.

[64] A. Noubigh, M. Abderrabba, E. Provost, Salt addition effect on partition coef- ficient of some phenolic compounds constituents of olive mill wastewater in 1-octanol-water system at 298.15K, J. Iran. Chem. Soc. 6 (2009) 168-176.

[65] Y.H. Tsai, K.F. Lee, Y. Bin Huang, C. Te Huang, P.C. Wu, In vitro permeation and in vivo whitening effect of topical hesperetin microemulsion delivery system, Int. J. Pharm. 388 (2010) 257-262.

[66] L.J. Jin, Z. Wei, J.Y. Dai, P. Guo, L.S. Wang, Prediction of partitioning properties for benzaldehydes by various molecular descriptors, Bull. Environ. Contam. Toxicol. 61 (1998) 1-7.

[67] D.M. Miller, Evidence that interfacial transport is rate-limiting during passive cell membrane permeation, BBA - Biomembr 1065 (1991) 75-81.

[68] A. Berthod, S. Ignatova, I.A. Sutherland, Advantages of a small-volume counter-current chromatography column, J. Chromatogr. A. 1216 (2009) 4169-4175.

[69] Z. Yang, P. Guo, R. Han, D. Wu, J.M. Gao, S. Wu, Methanol linear gradient counter-current chromatography for the separation of natural products: sinopodophyllum hexandrum as samples, J. Chromatogr. A. 1603 (2019) $251-261$.

[70] K. Srinivas, J.W. King, L.R. Howard, J.K. Monrad, Solubility and solution thermodynamic properties of quercetin and quercetin dihydrate in subcritical water, J. Food Eng. 100 (2010) 208-218.

[71] D. Althans, P. Schrader, S. Enders, Solubilisation of quercetin: comparison of hyperbranched polymer and hydrogel, J. Mol. Liq. 196 (2014) 86-93.

[72] J.C. Dearden, G.M. Bresnen, The measurement of partition coefficients, Quant. Struct. Relationships. 7 (1988) 133-144.

[73] C. Wittekindt, A. Klamt, COSMO-RS as a predictive tool for lipophilicity, QSAR Comb. Sci. 28 (2009) 874-877.

[74] S. Marsden-Jones, N. Colclough, I. Garrard, N. Sumner, S. Ignatova, Using quantitative structure activity relationship models to predict an appropriate solvent system from a common solvent system family for countercurrent chromatography separation, J. Chromatogr. A. 1398 (2015) 66-72.

[75] F. Shakeel, M. Salem-Bekhit, N. Haq, N.A. Siddiqui, Solubility and thermodynamics of ferulic acid in different neat solvents: measurement, correlation and molecular interactions, J. Mol. Liq. 236 (2017) 144-150.

[76] R.G. Bitencourt, F.A. Cabral, A.J.A. Meirelles, Ferulic acid solubility in supercritical carbon dioxide, ethanol and water mixtures, J. Chem. Thermodyn. 103 (2016) 285-291.

[77] N. Haq, N.A. Siddiqui, F. Shakeel, Solubility and molecular interactions of ferulic acid in various (isopropanol + water) mixtures, J. Pharm. Pharmacol. 69 (2017) 1485-1494.

[78] C. Zhou, X. Shi, H. Wang, N. An, Measurement and correlation of solubilities of trans-ferulic acid in different solvents, J. Chem. Ind. Eng. 58 (2007) 2705-2709.

[79] L. Liu, J. Chen, Solubility of hesperetin in various solvents from (288.2 to 323.2) K, J. Chem. Eng. Data. 53 (2008) 1649-1650.

[80] H. Zhang, M. Wang, L. Chen, Y. Liu, H. Liu, H. Huo, L. Sun, X. Ren, Y. Deng, A. Qi, Structure-solubility relationships and thermodynamic aspects of solubility of some flavonoids in the solvents modeling biological media, J. Mol. Liq. 225 (2017) 439-445

[81] A. Blahut, V. Dohnal, P. Vrbka, Interactions of volatile organic compounds with the ionic liquid 1-ethyl-3-methylimidazolium tetracyanoborate, J. Chem. Thermodyn. 47 (2012) 100-108.

[82] S. Griffin, S.G. Wyllie, J. Markham, Determination of octanol-water partition coefficient for terpenoids using reversed-phase high-performance liquid chromatography, J. Chromatogr. A. 864 (1999) 221-228.

[83] H. Ahmed, C.F. Poole, Model for the distribution of neutral organic compounds between n-hexane and acetonitrile, J. Chromatogr. A. 1104 (2006) 82-90.

[84] A.H. Elhamirad, M.H. Zamanipoor, Thermal stability of some flavonoids and phenolic acids in sheep tallow olein, Eur. J. Lipid Sci. Technol. 114 (2012) 602-606.

[85] N.A. Santos, A.M.T.M. Cordeiro, S.S. Damasceno, R.T. Aguiar, R. Rosenhaim, J.R. Carvalho Filho, I.M.G. Santos, A.S. Maia, A.G. Souza, Commercial antioxidants and thermal stability evaluations, Fuel 97 (2012) 638-643.

[86] S. Ignatova, P. Hewitson, B. Mathews, I. Sutherland, Evaluation of dual flow counter-current chromatography and intermittent counter-current extraction, J. Chromatogr. A. 1218 (2011) 6102-6106.

[87] A. Berthod, B. Billardello, S. Geoffroy, Polyphenols in countercurrent chromatography. An example of large scale separation, Analusis 27 (1999) 750-757.

[88] Z. Yang, Y. Wu, S. Wu, A combination strategy for extraction and isolation of multi-component natural products by systematic two-phase solvent extraction-13C nuclear magnetic resonance pattern recognition and following conical counter-current chromatography separation: podophyl, J. Chromatogr. A. 1431 (2016) 184-196.

[89] Y.I. Korenman, Distribution Ratios of Organic Compounds, Voronej (Russia), 1992.

[90] K.L. Kaygorodov, Y.V. Chelbina, V.E. Tarabanko, N.V. Tarabanko, Extraction of vanillin by aliphatic alcohols, J. Sib. Fed. Univ. 3 (2010) 228-233.

[91] M.I.F. Mota, P.C.R. Pinto, J.M. Loureiro, A.E. Rodrigues, Recovery of vanillin and syringaldehyde from lignin oxidation: a review of separation and purification processes, Sep. Purif. Rev. 45 (2016) 227-259.

[92] V.E. Tarabanko, N.M. Ivanchenko, A.V. Kudryashev, G.R. Gulbis, B.N. Kuznetsov, Method for extraction of vanillin, Russian Patent. - No 2065434.-20, 1996.

[93] Y. Lu, A. Berthod, R. Hu, W. Ma, Y. Pan, Screening of complex natural extracts by countercurrent chromatography using a parallel protocol, Anal. Chem. 81 (2009) 4048-4059.

[94] I. Sutherland, P. Hewitson, S. Ignatova, New 18-1 process-scale counter-current chromatography centrifuge, J. Chromatogr. A. 1216 (2009) 4201-4205.

[95] G. Hacer, I.I.R. Baptista, P.L. Wood, A. Livingston, A novel approach to modelling counter-current chromatography, J. Chromatogr. A. 1217 (2010) 6230-6240. 
[96] A. Berthod, K. Faure, Separations with a liquid stationary phase: countercurrent chromatography or centrifugal partition chromatography, Anal. Sep. Sci. 4 (2015) 1177-1206.

[97] P. Hewitson, Intermittent Counter-Current extraction: A new Continuous Dynamic Liquid-Liquid Extraction Methodology, Brunel University, 2014.
[98] J.B. Friesen, S. Ahmed, G.F. Pauli, Qualitative and quantitative evaluation of solvent systems for countercurrent separation, J. Chromatogr. A. 1377 (2015) 55-63. 